\title{
Homeomorphism metric space and the fixed point theorems
}

\author{
Yinglin Luo, Yongfu Su*, Wenbiao Gao \\ Department of Mathematics, Tianjin Polytechnic University, Tianjin 300387, China.
}

Communicated by R. Saadati

\begin{abstract}
The purpose of this paper is to introduce the concept of the homeomorphism metric space and to prove the fixed point theorems and the best proximity point theorems for generalized contractions in such spaces. The multiplicative metric space is a special form of the homeomorphism metric space. The results of this paper improve and extend the previously known ones in the literature. (C)2017 All rights reserved.
\end{abstract}

Keywords: Homeomorphism metric space, multiplicative metric space, metric space, b-metric space, generalized contraction, fixed point, best proximity point.

2010 MSC: 47H05, 47H09, 47H10.

\section{Introduction}

In 2008, Bashirov et al. [2] introduced the notion of multiplicative metric spaces and illustrated the usefulness of multiplicative calculus with some interesting applications. From then on, several authors have reported some important fixed point results in the framework of multiplicative metric spaces (see [2, 3, 5-7]). In 2016, Agarwal et al. [1] pointed out the relation between the multiplicative metric spaces and the standard metric spaces.

In this paper we introduce the concept of the homeomorphism metric space and prove the fixed point theorems and the best proximity point theorems for generalized contractions in such spaces. The concept of the homeomorphism metric space is firstly introduced in this article. It is worth noting that the multiplicative metric space is a special form of the homeomorphism metric space. The results of this paper improve and extend the previously known ones in the literature.

Now, we present some necessary definitions and results which will be needed in the article.

In 2008, Bashirov et al. [2] introduced the following notion of multiplicative metric spaces.

Definition 1.1 ([2]). Let $X$ be a non-empty set. A mapping $d^{*}: X \times X \rightarrow[0,+\infty)$ is said to be a multiplicative metric if it satisfies the following conditions:

\footnotetext{
*Corresponding author Gao)

Email addresses: tjluoyinglin@sina.com (Yinglin Luo), t jsuyongfu@163.com (Yongfu Su), 15822752271@163.com (Wenbiao

doi:10.22436/jnsa.010.10.01
} 
(i) $d^{*}(x, y) \geqslant 1$ for all $x, y \in X$;

(ii) $d^{*}(x, y)=1$ if and only if $x=y$;

(iii) $d^{*}(x, y)=d^{*}(y, x)$ for all $x, y \in X$;

(iv) $d^{*}(x, z) \leqslant d^{*}(x, z) \cdot d^{*}(z, y)$ for all $x, y, z \in X$.

Also, $\left(X, d^{*}\right)$ is called a multiplicative metric space.

The concept of b-metric spaces was introduced by Czerwik [4] in 1993, who used it to prove a generalization of Banach principle in spaces endowed with such kind of metrics.

Definition 1.2 ([4]). Let $X$ be a non-empty set. A mapping $d: X \times X \rightarrow[0,+\infty)$ is said to be a b-metric if it satisfies the following conditions:

(i) $d(x, y) \geqslant 0$ for all $x, y \in X$;

(ii) $d(x, y)=0$ if and only if $x=y$;

(iii) $d(x, y)=d(y, x)$ for all $x, y \in X$;

(iv) $d(x, y) \leqslant b d(y, z)+b d(z, y)$ for all $x, y, z \in X$,

where $b \geqslant 1$ is a constant. Also, $(X, d)$ is called a $b$-metric space.

In 2016, Agarwal et al. [1] pointed out the following relation.

Theorem $1.3([1])$. Let $\left(X, \mathrm{~d}^{*}\right)$ be a multiplicative metric space. Then the mapping $\mathrm{d}: \mathrm{X} \times \mathrm{X} \rightarrow[0,+\infty)$ with $\mathrm{d}(\mathrm{x}, \mathrm{y})=\ln \left(\mathrm{d}^{*}(\mathrm{x}, \mathrm{y})\right)$ forms a metric.

In 2015, Su and Yao [8] proved the existence and uniqueness of the fixed point for the generalized contraction type mappings in complete metric spaces.

Theorem 1.4 ([8]). Let $(\mathrm{X}, \mathrm{d})$ be a complete metric space, $\mathrm{T}: \mathrm{X} \rightarrow \mathrm{X}$ be a mapping such that

$$
\psi(d(T x, T y)) \leqslant \phi(d(x, y)), \forall x, y \in X,
$$

where $\psi, \phi:[0,+\infty) \rightarrow[0,+\infty)$ are two functions satisfying the conditions:

(1) $\psi(a) \leqslant \phi(b) \Rightarrow a \leqslant b$;

(2) $\left\{\begin{array}{l}\psi\left(a_{n}\right) \leqslant \phi\left(b_{n}\right) \\ a_{n} \rightarrow \varepsilon, b_{n} \rightarrow \varepsilon\end{array} \Rightarrow \varepsilon=0\right.$.

Then $\mathrm{T}$ has a unique fixed point and, for any given $\mathrm{x}_{0} \in \mathrm{X}$, the iterative sequence $\mathrm{T}^{\mathrm{n}} \mathrm{x}_{0}$ converges to this fixed point.

Example 1.5 ([8]). There are some functions that satisfy conditions (1) and (2) of Theorem 1.4.

(1) $\left\{\begin{array}{l}\psi_{1}(t)=t, \\ \phi_{1}(t)=\alpha t,\end{array}\right.$ where $0<\alpha<1$ is a constant;

(2) $\left\{\begin{array}{l}\psi_{2}(t)=t^{2} \\ \phi_{2}(t)=\ln \left(t^{2}+1\right)\end{array}\right.$

(3) $\left\{\begin{array}{l}\psi_{3}(t)=t, \\ \phi_{3}(t)= \begin{cases}t^{2}, & 0 \leqslant t \leqslant \frac{1}{2}, \\ t-\frac{3}{8}, & \frac{1}{2}<t<+\infty\end{cases} \end{array}\right.$

(4) $\left\{\begin{array}{l}\psi_{4}(t)= \begin{cases}t, & 0 \leqslant t \leqslant 1, \\ t-\frac{1}{2}, & 1<t<+\infty,\end{cases} \\ \phi_{4}(t)= \begin{cases}\frac{t}{2}, & 0 \leqslant t \leqslant 1, \\ t-\frac{4}{5}, & 1<t<+\infty ;\end{cases} \end{array}\right.$

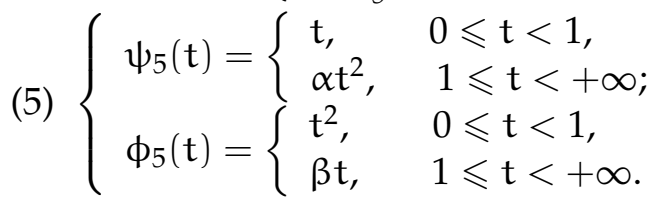


In 1969, Fan [5] introduced and established a classical best approximation theorem which is regarded as a natural generalization of fixed point theorems. Let $(A, B)$ be a pair of nonempty subsets of a metric space $(X, d)$, consider a mapping $T: A \rightarrow B$. The best proximity point problem is whether we can find an element $x_{0}$ in $A$ such that $d\left(x_{0}, T x_{0}\right)=\min \{d(x, T x): x \in A\}$. In fact, if $A=B$, then $d(A, B)=0$ and hence the best proximity point of $T$ becomes a fixed point of $T$. Since $d(x, T x) \geqslant d(A, B)$ for any $x \in A$, the optimal solution to this problem is the one for which the value $d(A, B)$ is attained. A point $x$ in $A$ for which $d(x, T x)=d(A, B)$ is called a best proximity point of $T$. We denote the following sets by $A_{0}$ and $B_{0}$,

$$
\begin{aligned}
& A_{0}=\{x \in A: d(x, y)=d(A, B) \text { for some } y \in B\}, \\
& B_{0}=\{y \in B: d(x, y)=d(A, B) \text { for some } x \in A\},
\end{aligned}
$$

where $d(A, B)=\inf \{d(x, y): x \in A$ and $y \in B\}$.

It is interesting that $A_{0}$ and $B_{0}$ are contained in the boundaries of $A$ and $B$ respectively provided $A$ and $B$ are closed subsets of a normed linear space such that $d(A, B)>0([9,10])$.

The following are some necessary definitions and results about the best proximity point theorems in metric space, which will be needed in our discussion.

Definition $1.6([9,10])$. Let $(A, B)$ be a pair of nonempty subsets of a metric space $(X, d)$ with $A_{0} \neq \emptyset$. Then the pair $(A, B)$ is said to have the P-property if and only if for any $x_{1}, x_{2} \in A_{0}$ and $y_{1}, y_{2} \in B_{0}$,

$$
\left\{\begin{array}{l}
d\left(x_{1}, y_{1}\right)=d(A, B) \\
d\left(x_{2}, y_{2}\right)=d(A, B)
\end{array} \Rightarrow d\left(x_{1}, x_{2}\right)=d\left(y_{1}, y_{2}\right)\right.
$$

Definition $1.7([9,10])$. Let $(A, B)$ be a pair of nonempty subsets of a metric space $(X, d)$ with $A_{0} \neq \emptyset$. Then the pair $(A, B)$ is said to have the weak P-property if and only if for any $x_{1}, x_{2} \in A_{0}$ and $y_{1}, y_{2} \in B_{0}$,

$$
\left\{\begin{array}{l}
d\left(x_{1}, y_{1}\right)=d(A, B) \\
d\left(x_{2}, y_{2}\right)=d(A, B)
\end{array} \Rightarrow d\left(x_{1}, x_{2}\right) \leqslant d\left(y_{1}, y_{2}\right)\right.
$$

Example $1.8([9])$. Consider $\left(R^{2}, d\right)$, where $d$ is the Euclidean distance and the subsets $A=\{(0,0)\}$ and $B=\left\{y=1+\sqrt{1-x^{2}}\right\}$. Obviously, $A_{0}=\{(0,0)\}, B_{0}=\{(-1,1),(1,1)\}$, and $d(A, B)=\sqrt{2}$. Furthermore,

$$
\mathrm{d}((0,0),(-1,1))=\mathrm{d}((0,0),(1,1))=\sqrt{2} ;
$$

however,

$$
0=\mathrm{d}((0,0),(0,0))<\mathrm{d}((-1,1),(1,1))=2 .
$$

We can see that the pair $(A, B)$ satisfies the weak P-property but not the P-property.

Definition 1.9 ([8]). Let $(A, B)$ be a pair of nonempty subsets of a metric space $(X, d)$ with $A_{0} \neq \emptyset$. Then the pair $(A, B)$ is said to have the $(\psi, \varphi)$-P-property if and only if for any $x_{1}, x_{2} \in A_{0}$ and $y_{1}, y_{2} \in B_{0}$,

$$
\left\{\begin{array}{l}
d\left(x_{1}, y_{1}\right)=d(A, B) \\
d\left(x_{2}, y_{2}\right)=d(A, B)
\end{array} \Rightarrow \psi\left(d\left(x_{1}, x_{2}\right)\right) \leqslant \varphi\left(d\left(y_{1}, y_{2}\right)\right)\right.
$$

where $\psi, \varphi:[0,+\infty) \rightarrow[0,+\infty)$ are two functions.

In 2015, Su and Yao [8] also proved the following best proximity point theorems for the generalized contraction type mappings in complete metric spaces.

Theorem 1.10 ([8]). Let $(A, B)$ be a pair of nonempty closed subsets of a complete metric space $(X, d)$ such that $A_{0} \neq \emptyset$. Let $\psi, \varphi, \phi:[0,+\infty) \rightarrow[0,+\infty)$ be three functions satisfying the conditions:

(1) $\psi(a) \leqslant \phi(b) \Rightarrow a \leqslant b ;$ 
(2) $\left\{\begin{array}{l}\psi\left(a_{n}\right) \leqslant \phi\left(b_{n}\right) \\ a_{n} \rightarrow \varepsilon, b_{n} \rightarrow \varepsilon\end{array} \Rightarrow \varepsilon=0\right.$

(3) $\psi\left(t_{n}\right) \rightarrow 0 \Rightarrow t_{n} \rightarrow 0$;

(4) $t_{n} \rightarrow 0 \Rightarrow \varphi\left(t_{n}\right) \rightarrow 0$;

(5) $\varphi(a) \leqslant \varphi(b) \Rightarrow a \leqslant b$.

Let $\mathrm{T}: \mathrm{A} \rightarrow \mathrm{B}$ be a mapping, such that

$$
\psi(d(T x, T y)) \leqslant \phi(d(x, y)), \quad \forall x, y \in A .
$$

Suppose that the pair $(\mathrm{A}, \mathrm{B})$ has the $(\psi, \varphi)$-P-property and $\mathrm{T}\left(\mathrm{A}_{0}\right) \subseteq \mathrm{B}_{0}$. Then there exists a unique $\mathrm{x}^{*} \in \mathrm{A}$ such that $\mathrm{d}\left(\mathrm{x}^{*}, \mathrm{~T} x^{*}\right)=\mathrm{d}(\mathrm{A}, \mathrm{B})$.

Theorem 1.11 ([8]). Let (A, B) be a pair of nonempty closed subsets of a complete metric space $(\mathrm{X}, \mathrm{d})$ such that $A_{0} \neq \emptyset$. Let $\psi, \phi:[0,+\infty) \rightarrow[0,+\infty)$ be two functions satisfying the conditions:

(1) $\psi(a) \leqslant \phi(b) \Rightarrow a \leqslant b$;

(2) $\left\{\begin{array}{l}\psi\left(a_{n}\right) \leqslant \phi\left(b_{n}\right) \\ a_{n} \rightarrow \varepsilon, b_{n} \rightarrow \varepsilon\end{array} \Rightarrow \varepsilon=0\right.$;

(3) $\psi\left(t_{n}\right) \rightarrow 0 \Leftrightarrow t_{n} \rightarrow 0$;

and $\psi(\mathrm{t})$ is nondecreasing. Let $\mathrm{T}: \mathrm{A} \rightarrow \mathrm{B}$ be a mapping such that

$$
\psi(d(T x, T y)) \leqslant \phi(d(x, y)), \forall x, y \in A .
$$

Suppose that the pair $(A, B)$ has the weak P-property and $\mathrm{T}\left(\mathrm{A}_{0}\right) \subseteq \mathrm{B}_{0}$. Then there exists a unique $\mathrm{x}^{*} \in \mathrm{A}$ such that $\mathrm{d}\left(\mathrm{x}^{*}, T x^{*}\right)=\mathrm{d}(\mathrm{A}, \mathrm{B})$.

\section{Homeomorphism metric space and the fixed point theorems}

We start our work by introducing the following concepts. The concept of homeomorphism metric space was firstly introduced in this paper.

Definition 2.1. Let $X$ be a non-empty set. A mapping $d^{*}: X \times X \rightarrow[0,+\infty)$ is said to be a metric embryo if it satisfies the following conditions:

(i) $d^{*}(x, y) \geqslant a$ for all $x, y \in X$;

(ii) $d^{*}(x, y)=a$ if and only if $x=y$;

(iii) $d^{*}(x, y)=d^{*}(y, x)$ for all $x, y \in X$,

where $a \in[0,+\infty)$ is a constant. Also, $\left(X, d^{*}\right)$ is called a metric embryo space.

Definition 2.2. A metric embryo space $\left(X, d^{*}\right)$ is said to be a homeomorphism metric space, if there exists a continuous and strictly increasing function $f:[0,+\infty) \rightarrow[a,+\infty)$ with $\lim _{t \rightarrow+\infty} f(t)=+\infty$ such that

$$
\mathrm{d}(x, y)=\mathrm{f}^{-1}\left(\mathrm{~d}^{*}(x, y)\right), \quad \forall x, y \in X
$$

forms a metric. We denote $\left(X, d^{*}\right)=\left(X, d^{*}, f\right)$. In this case, $d^{*}(x, y)=f(d(x, y))$ for all $x, y \in X$.

The basic topological structure of homeomorphism metric space $\left(X, d^{*}, f\right)$ is consistent with the metric space $(X, d)$. For examples, the open set, closed set, completeness, Cauchy sequence, convergence, continuity, compact set and so on.

Definition 2.3. A metric embryo space $\left(X, d^{*}\right)$ is said to be a homeomorphism b-metric space, if there exists a continuous and strictly increasing function $f:[0,+\infty) \rightarrow[a,+\infty)$ with $\lim _{t \rightarrow+\infty} f(t)=+\infty$ such that

$$
d(x, y)=f^{-1}\left(d^{*}(x, y)\right), \quad \forall x, y \in X
$$

forms a b-metric. We denote $\left(X, d^{*}\right)=\left(X, d^{*}, f\right)$. In this case, $d^{*}(x, y)=f(d(x, y))$ for all $x, y \in X$. 
The basic topological structure of homeomorphism $b$-metric space $\left(X, d^{*}, f\right)$ is consistent with the $b$ metric space $(X, d)$. For examples, the open set, closed set, completeness, Cauchy sequence, convergence, continuity, compact set and so on.

Example 2.4. Let $\left(X, d^{*}\right)$ be a multiplicative metric space, then $\left(X, d^{*}\right)$ must be a homeomorphism metric space $\left(X, d^{*}, f\right)$ with $f(t)=e^{t}, t \in[0,+\infty)$.

Theorem 2.5. Let $\left(\mathrm{X}, \mathrm{d}^{*}\right)$ be a b-metric space, then $\left(\mathrm{X}, \mathrm{d}^{*}\right)$ is also a homeomorphism $\sqrt[2 n]{\mathrm{b}}$-metric space with $\mathrm{f}(\mathrm{t})=\mathrm{t}^{2 \mathrm{n}}, \mathrm{t} \in[0,+\infty)$, where $\mathrm{n}$ is a natural number.

Proof. Let $\left(X, \mathrm{~d}^{*}\right)$ be a b-metric space, $\mathrm{f}(\mathrm{t})=\mathrm{t}^{2}$, and

$$
\mathrm{d}(x, y)=\sqrt{\mathrm{d}^{*}(x, y)}, \forall x, y \in X
$$

We have for all $x, y, z \in X$ that

$$
d^{2}(x, y)=d^{*}(x, y) \leqslant b d^{*}(x, z)+b d^{*}(z, y) \leqslant b d^{2}(x, z)+b d^{2}(z, y) \leqslant(\sqrt{b} d(x, z)+\sqrt{b} d(z, y))^{2}
$$

This implies for all $x, y, z \in X$ that

$$
d(x, y) \leqslant \sqrt{b} d(x, z)+\sqrt{b} d(z, y)
$$

By induction, let $f(t)=t^{2 n}$ and

$$
d(x, y)=\sqrt[2 n]{d^{*}(x, y)}, \forall x, y \in X
$$

We have for all $x, y, z \in X$ that

$$
d(x, y) \leqslant \sqrt[2 n]{b} d(x, z)+\sqrt[2 n]{b} d(z, y)
$$

This completes the proof.

Theorem 2.6. Let $\left(\mathrm{X}, \mathrm{d}^{*}\right)$ be a complete multiplicative metric space, and $\mathrm{T}: \mathrm{X} \rightarrow \mathrm{X}$ be a mapping such that

$$
\psi\left(d^{*}(T x, T y)\right) \leqslant \phi\left(d^{*}(x, y)\right), \quad \forall x, y \in X,
$$

where $\psi, \phi:[0,+\infty) \rightarrow[0,+\infty)$ are two functions satisfying the conditions:

(1) $\psi(a) \leqslant \phi(b) \Rightarrow a \leqslant b$;

(2) $\left\{\begin{array}{l}\psi\left(a_{n}\right) \leqslant \phi\left(b_{n}\right) \\ a_{n} \rightarrow \varepsilon, b_{n} \rightarrow \varepsilon\end{array} \Rightarrow \varepsilon=1\right.$.

Then $\mathrm{T}$ has a unique fixed point and, for any given $\mathrm{x}_{0} \in \mathrm{X}$, the iterative sequence $\mathrm{T}^{\mathrm{n}} \mathrm{x}_{0}$ converges to this fixed point.

Proof. The condition (2.1) can be rewritten as

$$
\psi\left(e^{\ln d^{*}(T x, T y)}\right) \leqslant \phi\left(e^{\ln d^{*}(x, y)}\right), \quad \forall x, y \in X .
$$

Let $\psi^{*}(t)=\psi\left(e^{t}\right), \phi^{*}(t)=\phi\left(e^{t}\right)$, then above inequality becomes that

$$
\psi^{*}\left(\ln d^{*}(T x, T y)\right) \leqslant \phi^{*}\left(\ln d^{*}(x, y)\right), \quad \forall x, y \in X .
$$

From the conditions (1) and (2), we have the following conditions

$\left(1^{\prime}\right) \psi^{*}(a) \leqslant \phi^{*}(b) \Rightarrow a \leqslant b ;$

$\left(2^{\prime}\right)\left\{\begin{array}{l}\psi^{*}\left(a_{n}\right) \leqslant \phi^{*}\left(b_{n}\right) \\ a_{n} \rightarrow \varepsilon, b_{n} \rightarrow \varepsilon\end{array} \Rightarrow \varepsilon=0\right.$.

Since $\ln d^{*}(x, y)$ is a metric on $X$, consider the condition (2.2), $\left(1^{\prime}\right)$ and $\left(2^{\prime}\right)$, by using Theorem 1.4 , we can get the conclusion of Theorem 2.6. This completes the proof. 
Theorem 2.7. Let $\left(\mathrm{X}, \mathrm{d}^{*}, \mathrm{f}\right)$ be a complete homeomorphism metric space, and $\mathrm{T}: \mathrm{X} \rightarrow \mathrm{X}$ be a mapping such that

$$
\psi\left(d^{*}(T x, T y)\right) \leqslant \phi\left(d^{*}(x, y)\right), \quad \forall x, y \in X,
$$

where $\psi, \phi:[0,+\infty) \rightarrow[0,+\infty)$ are two functions satisfying the conditions:

(1) $\psi(a) \leqslant \phi(b) \Rightarrow a \leqslant b$;

(2) $\left\{\begin{array}{l}\psi\left(a_{n}\right) \leqslant \phi\left(b_{n}\right) \\ a_{n} \rightarrow \varepsilon, b_{n} \rightarrow \varepsilon\end{array} \Rightarrow \varepsilon=f(0)\right.$

Then $\mathrm{T}$ has a unique fixed point and, for any given $\mathrm{x}_{0} \in \mathrm{X}$, the iterative sequence $\mathrm{T}^{\mathrm{n}} \mathrm{x}_{0}$ converges to this fixed point.

Proof. From (2.3), we have that

$$
\psi(f(d(T x, T y))) \leqslant \phi(f(d(x, y))), \quad \forall x, y \in X,
$$

which can be rewritten as

$$
(\psi f)(d(T x, T y)) \leqslant(\phi f)(d(x, y)), \quad \forall x, y \in X .
$$

Let $\psi_{\mathrm{f}}=\psi \cdot f, \phi_{\mathrm{f}}=\phi \cdot f$, then $(2.4)$ can be rewritten as

$$
\psi_{f}(d(T x, T y)) \leqslant \phi_{f}(d(x, y)), \quad \forall x, y \in X
$$

Next, we show that, the functions $\psi_{f}$ and $\phi_{f}$ satisfy the conditions (1) and (2) as in Theorem 1.4. In fact, if

$$
\psi_{f}(a) \leqslant \phi_{f}(b)
$$

that is,

$$
\psi(f(a)) \leqslant \phi(f(b)),
$$

we have from the condition (1) as in Theorem 1.4, that $f(a) \leqslant f(b)$, therefore, it follows from the monotonicity of $f$, that $a \leqslant b$. We have proved the functions $\psi_{f}$ and $\phi_{f}$ satisfy the condition (1) as in Theorem 1.4. Furthermore, if

$$
\left\{\begin{array}{l}
\psi_{f}\left(a_{n}\right) \leqslant \phi_{f}\left(b_{n}\right) \\
a_{n} \rightarrow \varepsilon, b_{n} \rightarrow \varepsilon
\end{array}\right.
$$

that is,

$$
\left\{\begin{array}{l}
\psi\left(f\left(a_{n}\right)\right) \leqslant \phi\left(f\left(b_{n}\right)\right), \\
f\left(a_{n}\right) \rightarrow f(\varepsilon), f\left(b_{n}\right) \rightarrow f(\varepsilon),
\end{array}\right.
$$

we have from the condition (2) as in Theorem 1.4, that $f(\varepsilon)=f(0)$, therefore, it follows from the continuity of $f$, that $\varepsilon=0$.

Now, we have proved the functions $\psi_{f}$ and $\phi_{f}$ satisfy the conditions (1) and (2) as in Theorem 1.4. Consider the inequality (2.5), by using Theorem 1.4, we get the conclusion of Theorem 2.7. This completes the proof.

By using Theorem 1.4, we can get the following results.

Corollary 2.8. Let $\left(\mathrm{X}, \mathrm{d}^{*}, \mathrm{f}\right)$ be a complete homeomorphism metric space, and $\mathrm{T}: \mathrm{X} \rightarrow \mathrm{X}$ be a mapping such that

$$
\psi\left(e^{d(T x, T y)}\right) \leqslant \phi\left(e^{d(x, y)}\right), \quad \forall x, y \in X,
$$

where $\psi, \phi:[0,+\infty) \rightarrow[0,+\infty)$ are two functions satisfying the conditions:

(1) $\psi(a) \leqslant \phi(b) \Rightarrow a \leqslant b$;

(2) $\left\{\begin{array}{l}\psi\left(a_{n}\right) \leqslant \phi\left(b_{n}\right) \\ a_{n} \rightarrow \varepsilon, b_{n} \rightarrow \varepsilon\end{array} \Rightarrow \varepsilon=1\right.$. 
Then $\mathrm{T}$ has a unique fixed point and, for any given $\mathrm{x}_{0} \in \mathrm{X}$, the iterative sequence $\mathrm{T}^{\mathrm{n}} \mathrm{x}_{0}$ converges to this fixed point. Proof. Let $\mathrm{f}(\mathrm{t})=\mathrm{e}^{\mathrm{t}}$ in Theorem 1.4, we can get Corollary 2.8. This completes the proof.

Corollary 2.9. Let $\left(\mathrm{X}, \mathrm{d}^{*}, \mathrm{f}\right)$ be a complete homeomorphism metric space, and $\mathrm{T}: \mathrm{X} \rightarrow \mathrm{X}$ be a mapping such that

$$
\psi\left(d(T x, T y)^{l}\right) \leqslant \phi\left(d(x, y)^{l}\right), \quad \forall x, y \in X,
$$

where $l>0$ is a constant and $\psi, \phi:[0,+\infty) \rightarrow[0,+\infty)$ are two functions satisfying the conditions:

(1) $\psi(a) \leqslant \phi(b) \Rightarrow a \leqslant b$;

(2) $\left\{\begin{array}{l}\psi\left(a_{n}\right) \leqslant \phi\left(b_{n}\right) \\ a_{n} \rightarrow \varepsilon, b_{n} \rightarrow \varepsilon\end{array} \Rightarrow \varepsilon=0\right.$

Then $\mathrm{T}$ has a unique fixed point and, for any given $\mathrm{x}_{0} \in \mathrm{X}$, the iterative sequence $\mathrm{T}^{\mathrm{n}} \mathrm{x}_{0}$ converges to this fixed point.

Proof. Let $\mathrm{f}(\mathrm{t})=\mathrm{t}^{\mathrm{l}}$ in Theorem 1.4, we can get Corollary 2.9. This completes the proof.

Corollary 2.10. Let $\left(\mathrm{X}, \mathrm{d}^{*}, \mathrm{f}\right)$ be a complete homeomorphism metric space, and $\mathrm{T}: \mathrm{X} \rightarrow \mathrm{X}$ be a mapping such that

$$
\psi\left(\tan \frac{\pi \mathrm{d}(T x, T y)}{2+2 \mathrm{~d}(T x, T y)}\right) \leqslant \phi\left(\tan \frac{\pi \mathrm{d}(x, y)}{2+2 \mathrm{~d}(x, y)}\right), \forall x, y \in X,
$$

where $l>0$ is a constant and $\psi, \phi:[0,+\infty) \rightarrow[0,+\infty)$ are two functions satisfying the conditions:

(1) $\psi(a) \leqslant \phi(b) \Rightarrow a \leqslant b$;

(2) $\left\{\begin{array}{l}\psi\left(a_{n}\right) \leqslant \phi\left(b_{n}\right) \\ a_{n} \rightarrow \varepsilon, b_{n} \rightarrow \varepsilon\end{array} \Rightarrow \varepsilon=0\right.$.

Then $\mathrm{T}$ has a unique fixed point and, for any given $\mathrm{x}_{0} \in \mathrm{X}$, the iterative sequence $\mathrm{T}^{\mathrm{n}} \mathrm{x}_{0}$ converges to this fixed point.

Proof. Let $f(t)=\tan \frac{\pi t}{2+2 t}$ in Theorem 1.4, we can get Corollary 2.10. This completes the proof.

\section{Generalized best proximity point theorems in the homeomorphism metric space}

In this section we will prove the best proximity point theorems for generalized contractions in the homeomorphism metric space.

Theorem 3.1. Let $\left(X, \mathrm{~d}^{*}, \mathrm{f}\right)$ be a complete homeomorphism metric space and $(\mathrm{A}, \mathrm{B})$ be a pair closed subset of $\mathrm{X}$ such that $A_{0} \neq \emptyset$. Let $\psi, \varphi, \phi:[0,+\infty) \rightarrow[0,+\infty)$ be three functions satisfying the conditions:

(1) $\psi(a) \leqslant \phi(b) \Rightarrow a \leqslant b$;

(2) $\left\{\begin{array}{l}\psi\left(a_{n}\right) \leqslant \phi\left(b_{n}\right) \\ a_{n} \rightarrow \varepsilon, b_{n} \rightarrow \varepsilon\end{array} \Rightarrow \varepsilon=f(0)\right.$;

(3) $\psi\left(t_{n}\right) \rightarrow 0 \Rightarrow t_{n} \rightarrow f(0)$;

(4) $t_{n} \rightarrow f(0) \Rightarrow \varphi\left(t_{n}\right) \rightarrow 0$;

(5) $\varphi(a) \leqslant \phi(b) \Rightarrow a \leqslant b$.

Let $\mathrm{T}: \mathrm{A} \rightarrow \mathrm{B}$ be a mapping, such that

$$
\psi\left(d^{*}(T x, T y)\right) \leqslant \phi\left(d^{*}(x, y)\right), \quad \forall x, y \in A .
$$

Suppose that the pair $(A, B)$ has the $(\psi, \varphi)$-P-property and $\mathrm{T}\left(\mathrm{A}_{0}\right) \subseteq \mathrm{B}_{0}$. Then there exists a unique $\mathrm{x}^{*} \in \mathrm{A}$ such that $\mathrm{d}^{*}\left(\mathrm{x}^{*}, T x^{*}\right)=\mathrm{d}^{*}(\mathrm{~A}, \mathrm{~B})$.

Proof. From (3.1), we have that

$$
\psi(f(d(T x, T y))) \leqslant \phi(f(d(x, y))), \quad \forall x, y \in X,
$$

which can be rewritten as 


$$
(\psi f)(d(T x, T y)) \leqslant(\phi f)(d(x, y)), \quad \forall x, y \in X .
$$

Let $\psi_{\mathrm{f}}=\psi \cdot f, \phi_{\mathrm{f}}=\phi \cdot \mathrm{f}$, then (3.2) can be rewritten as

$$
\psi_{f}(d(T x, T y)) \leqslant \phi_{f}(d(x, y)), \quad \forall x, y \in X
$$

Next, we show that, the functions $\psi_{f}$ and $\phi_{f}$ satisfy the conditions as in Theorem 1.10. In fact, if

$$
\psi_{f}(a) \leqslant \phi_{f}(b)
$$

that is,

$$
\psi(f(a)) \leqslant \phi(f(b)),
$$

we have from the condition (1) as in Theorem 1.10 that $f(a) \leqslant f(b)$, therefore, it follows from the monotonicity of $f$, that $a \leqslant b$. We have proved the functions $\psi_{f}$ and $\phi_{f}$ satisfy the condition (1) as in Theorem 1.10. Similarly, we can get the condition (5) as in Theorem 1.10. Furthermore, if

$$
\left\{\begin{array}{l}
\psi_{f}\left(a_{n}\right) \leqslant \phi_{f}\left(b_{n}\right) \\
a_{n} \rightarrow \varepsilon, b_{n} \rightarrow \varepsilon
\end{array}\right.
$$

that is,

$$
\left\{\begin{array}{l}
\psi\left(f\left(a_{n}\right)\right) \leqslant \phi\left(f\left(b_{n}\right)\right) \\
f\left(a_{n}\right) \rightarrow f(\varepsilon), f\left(b_{n}\right) \rightarrow f(\varepsilon),
\end{array}\right.
$$

we have from the condition (2) as in Theorem 1.10, that $f(\varepsilon)=f(0)$, therefore, it follows from the continuity of $f$, that $\varepsilon=0$.

Now, we show that, the functions $\psi_{f}$ and $\phi_{f}$ satisfy the conditions (3) and (4) in Theorem 1.10.

If $\psi_{f}\left(t_{n}\right)=\psi\left(f\left(t_{n}\right)\right) \rightarrow 0$, by the condition (3) in Theorem 3.1, we have $f\left(t_{n}\right) \rightarrow f(0)$, it follows from the continuity of $f$, that $t_{n} \rightarrow 0$. Then we get the condition (4) as in Theorem 1.10.

Let $\varphi_{f}\left(t_{n}\right)=\varphi\left(f\left(t_{n}\right)\right)$ and $t_{n} \rightarrow 0$, by continuity of $f$, that $f\left(t_{n}\right) \rightarrow f(0)$. From the condition (4) in Theorem 3.1 we can get that $\varphi_{f}\left(t_{n}\right)=\varphi\left(f\left(t_{n}\right)\right) \rightarrow 0$, then we have the condition (4) as in Theorem 1.10.

Taking into all the discussion offered above, the conditions in Theorem 3.1 can be rewritten as follows

$\left(1^{\prime}\right) \psi_{f}(a) \leqslant \phi_{f}(b) \Rightarrow a \leqslant b ;$

$\left(2^{\prime}\right)\left\{\begin{array}{l}\psi_{f}\left(a_{n}\right) \leqslant \phi_{f}\left(b_{n}\right) \\ a_{n} \rightarrow \varepsilon, b_{n} \rightarrow \varepsilon\end{array} \Rightarrow \varepsilon=0 ;\right.$

(3') $\psi_{\mathrm{f}}\left(\mathrm{t}_{\mathrm{n}}\right) \rightarrow 0 \Rightarrow \mathrm{t}_{\mathrm{n}} \rightarrow 0$;

$\left(4^{\prime}\right) t_{n} \rightarrow 0 \Rightarrow \varphi_{f}\left(t_{n}\right) \rightarrow 0$;

(5') $\varphi_{\mathrm{f}}(\mathrm{a}) \leqslant \phi_{\mathrm{f}}(\mathrm{b}) \Rightarrow \mathrm{a} \leqslant \mathrm{b}$.

Consider the condition (3.3) and all conditions offered above, by using Theorem 1.10, we can get the conclusion of Theorem 3.1. This completes the proof.

Theorem 3.2. Let $\left(X, \mathrm{~d}^{*}, \mathrm{f}\right)$ be a complete homeomorphism metric space and $(\mathrm{A}, \mathrm{B})$ be a pair closed subset of $\mathrm{X}$ such that $A_{0} \neq \emptyset$. Let $\psi, \phi:[0,+\infty) \rightarrow[0,+\infty)$ be two functions satisfying the conditions:

(1) $\psi(a) \leqslant \phi(b) \Rightarrow a \leqslant b$;

(2) $\left\{\begin{array}{l}\psi\left(a_{n}\right) \leqslant \phi\left(b_{n}\right) \\ a_{n} \rightarrow \varepsilon, b_{n} \rightarrow \varepsilon\end{array} \Rightarrow \varepsilon=f(0)\right.$;

(3) $\psi\left(\mathrm{t}_{\mathrm{n}}\right) \rightarrow 0 \Leftrightarrow \mathrm{t}_{\mathrm{n}} \rightarrow \mathrm{f}(0)$,

and $\psi(\mathrm{t})$ is nondecreasing. Let $\mathrm{T}: \mathrm{A} \rightarrow \mathrm{B}$ be a mapping such that

$$
\psi\left(d^{*}(T x, T y)\right) \leqslant \phi\left(d^{*}(x, y)\right), \quad \forall x, y \in A .
$$

Suppose that the pair $(A, B)$ has the weak P-property and $\mathrm{T}\left(\mathrm{A}_{0}\right) \subseteq \mathrm{B}_{0}$. Then there exists a unique $x^{*} \in \mathrm{A}$ such that $\mathrm{d}^{*}\left(\mathrm{x}^{*}, T x^{*}\right)=\mathrm{d}^{*}(\mathrm{~A}, \mathrm{~B})$. 
Proof. Let $\varphi(t)=\psi(t)$ for all $t \in[0,+\infty)$. Then the pair $(A, B)$ having the weak P-property implies that the pair $(A, B)$ has the $(\psi, \varphi)$-P-property. Condition (3) of Theorem 3.2 implies conditions (3), (4) of Theorem 3.1 and (3.4) implies (3.1). By using Theorem 3.1 we get the conclusion of Theorem 3.2.

Corollary 3.3. Let $\left(X, \mathrm{~d}^{*}\right)$ be a complete multiplicative metric space, $(\mathrm{A}, \mathrm{B})$ be a pair closed subset of $\mathrm{X}$ such that $A_{0} \neq \emptyset$, and $\psi, \varphi, \phi:[0,+\infty) \rightarrow[0,+\infty)$ are three functions satisfying the conditions:

(1) $\psi(a) \leqslant \phi(b) \Rightarrow a \leqslant b$;

(2) $\left\{\begin{array}{l}\psi\left(a_{n}\right) \leqslant \phi\left(b_{n}\right) \\ a_{n} \rightarrow \varepsilon, b_{n} \rightarrow \varepsilon\end{array} \Rightarrow \varepsilon=1\right.$;

(3) $\psi\left(t_{n}\right) \rightarrow 0 \Rightarrow t_{n} \rightarrow 1$;

(4) $t_{n} \rightarrow 1 \Rightarrow \varphi\left(t_{n}\right) \rightarrow 0$;

(5) $\varphi(a) \leqslant \phi(b) \Rightarrow a \leqslant b$.

Let $\mathrm{T}: \mathrm{A} \rightarrow \mathrm{B}$ be a mapping, such that

$$
\psi\left(d^{*}(T x, T y)\right) \leqslant \phi\left(d^{*}(x, y)\right), \quad \forall x, y \in A .
$$

Suppose that the pair $(A, B)$ has the $(\psi, \varphi)$-P-property and $\mathrm{T}\left(\mathrm{A}_{0}\right) \subseteq \mathrm{B}_{0}$. Then there exists a unique $x^{*} \in \mathrm{A}$ such that $\mathrm{d}^{*}\left(x^{*}, T x^{*}\right)=d^{*}(A, B)$.

Proof. Let $\mathrm{f}(\mathrm{t})=e^{\mathrm{t}}, \mathrm{t} \in[0,+\infty)$. Then a multiplicative metric space $\left(X, \mathrm{~d}^{*}\right)$ must be a homeomorphism metric space $\left(X, d^{*}, f\right)$. By using Theorem 3.1 we get the conclusion of Corollary 3.3.

Corollary 3.4. Let $\left(\mathrm{X}, \mathrm{d}^{*}\right)$ be a complete multiplicative metric space, $(\mathrm{A}, \mathrm{B})$ be a pair closed subset of $\mathrm{X}$ such that $A_{0} \neq \emptyset$, and $\psi, \phi:[0,+\infty) \rightarrow[0,+\infty)$ are two functions satisfying the conditions:

(1) $\psi(a) \leqslant \phi(b) \Rightarrow a \leqslant b$;

(2) $\left\{\begin{array}{l}\psi\left(a_{n}\right) \leqslant \phi\left(b_{n}\right) \\ a_{n} \rightarrow \varepsilon, b_{n} \rightarrow \varepsilon\end{array} \Rightarrow \varepsilon=1\right.$;

(3) $\psi\left(t_{\mathrm{n}}\right) \rightarrow 0 \Leftrightarrow t_{\mathrm{n}} \rightarrow 1$;

and $\psi(\mathrm{t})$ is nondecreasing. Let $\mathrm{T}: \mathrm{A} \rightarrow \mathrm{B}$ be a mapping, such that

$$
\psi\left(d^{*}(T x, T y)\right) \leqslant \phi\left(d^{*}(x, y)\right), \forall x, y \in A .
$$

Suppose that the pair $(A, B)$ has the weak P-property and $\mathrm{T}\left(\mathrm{A}_{0}\right) \subseteq \mathrm{B}_{0}$. Then there exists a unique $\mathrm{x}^{*} \in \mathrm{A}$ such that $\mathrm{d}^{*}\left(x^{*}, T x^{*}\right)=\mathrm{d}^{*}(\mathrm{~A}, \mathrm{~B})$.

Proof. Let $\varphi(t)=\psi(t)$ for all $t \in[0,+\infty)$. Then the pair $(A, B)$ having the weak P-property implies that the pair $(A, B)$ has the $(\psi, \varphi)$-P-property. Condition (3) of Corollary 3.4 implies conditions (3) and (4) of Corollary 3.3 and (3.6) implies (3.5). By using Corollary 3.3 we get the conclusion of Corollary 3.4.

Corollary 3.5. Let $\left(\mathrm{X}, \mathrm{d}^{*}\right)$ be a complete $\sqrt[2 n]{\mathrm{b}}$-metric space, $(\mathrm{A}, \mathrm{B})$ be a pair closed subset of $\mathrm{X}$ such that $\mathrm{A}_{0} \neq \emptyset$, and $\psi, \varphi, \phi:[0,+\infty) \rightarrow[0,+\infty)$ are three functions satisfying the conditions:

(1) $\psi(a) \leqslant \phi(b) \Rightarrow a \leqslant b ;$

(2) $\left\{\begin{array}{l}\psi\left(a_{n}\right) \leqslant \phi\left(b_{n}\right) \\ a_{n} \rightarrow \varepsilon, b_{n} \rightarrow \varepsilon\end{array} \Rightarrow \varepsilon=0\right.$

(3) $\psi\left(t_{n}\right) \rightarrow 0 \Rightarrow t_{n} \rightarrow 0$;

(4) $t_{n} \rightarrow 0 \Rightarrow \varphi\left(t_{n}\right) \rightarrow 0$;

(5) $\varphi(a) \leqslant \phi(b) \Rightarrow a \leqslant b$.

Let $\mathrm{T}: \mathrm{A} \rightarrow \mathrm{B}$ be a mapping, such that

$$
\psi\left(d^{*}(T x, T y)\right) \leqslant \phi\left(d^{*}(x, y)\right), \forall x, y \in A .
$$

Suppose that the pair $(A, B)$ has the $(\psi, \varphi)$-P-property and $\mathrm{T}\left(\mathrm{A}_{0}\right) \subseteq \mathrm{B}_{0}$. Then there exists a unique $\mathrm{x}^{*} \in \mathrm{A}$ such that $\mathrm{d}^{*}\left(\mathrm{x}^{*}, T \mathrm{x}^{*}\right)=\mathrm{d}^{*}(\mathrm{~A}, \mathrm{~B})$. 
Proof. Let $\mathrm{f}(\mathrm{t})=\mathrm{t}^{2 \mathrm{n}}, \mathrm{t} \in[0,+\infty)$. Then a $\sqrt[2 n]{\mathrm{b}}$-metric space $\left(X, \mathrm{~d}^{*}\right)$ must be a homeomorphism metric space $\left(X, d^{*}, f\right)$. By using Theorem 3.1 we get the conclusion of Corollary 3.5.

Corollary 3.6. Let $\left(\mathrm{X}, \mathrm{d}^{*}\right)$ be a complete $\sqrt[2 n]{\mathrm{b}}$-metric space, $(\mathrm{A}, \mathrm{B})$ be a pair closed subset of $\mathrm{X}$ such that $\mathrm{A}_{0} \neq \emptyset$, and $\psi, \phi:[0,+\infty) \rightarrow[0,+\infty)$ are two functions satisfying the conditions:

(1) $\psi(a) \leqslant \phi(b) \Rightarrow a \leqslant b$;

(2) $\left\{\begin{array}{l}\psi\left(a_{n}\right) \leqslant \phi\left(b_{n}\right) \\ a_{n} \rightarrow \varepsilon, b_{n} \rightarrow \varepsilon\end{array} \Rightarrow \varepsilon=0\right.$;

(3) $\psi\left(t_{n}\right) \rightarrow 0 \Leftrightarrow t_{n} \rightarrow 0$,

and $\psi(\mathrm{t})$ is nondecreasing. Let $\mathrm{T}: \mathrm{A} \rightarrow \mathrm{B}$ be a mapping, such that

$$
\psi\left(d^{*}(T x, T y)\right) \leqslant \phi\left(d^{*}(x, y)\right), \forall x, y \in A .
$$

Suppose that the pair $(\mathrm{A}, \mathrm{B})$ has the weak $\mathrm{P}$-property and $\mathrm{T}\left(\mathrm{A}_{0}\right) \subseteq \mathrm{B}_{0}$. Then there exists a unique $\mathrm{x}^{*} \in \mathrm{A}$ such that $d^{*}\left(x^{*}, T x^{*}\right)=d^{*}(A, B)$.

Proof. The process of proof is similar to the proof of Corollary 3.4. So we omit it here.

If we choose $\psi_{3}, \phi_{3}$ in Example 1.5, by Theorem 3.2, we can get the following result.

Corollary 3.7. Let $\left(\mathrm{X}, \mathrm{d}^{*}, \mathrm{f}\right)$ be a complete homeomorphism metric space, $(\mathrm{A}, \mathrm{B})$ be a pair closed subset of $\mathrm{X}$ such that $\mathrm{A}_{0} \neq \emptyset$. Let $\mathrm{T}: \mathrm{A} \rightarrow \mathrm{B}$ be a mapping such that

$$
\begin{aligned}
0 \leqslant & d^{*}(x, y) \leqslant \frac{1}{2} \Rightarrow d^{*}(T x, T y) \leqslant\left(d^{*}(x, y)\right)^{2}, \\
\frac{1}{2}<d^{*}(x, y) & \Rightarrow d^{*}(T x, T y) \leqslant d^{*}(x, y)-\frac{1}{8}
\end{aligned}
$$

for any $x, y \in A$. Suppose that the pair $(A, B)$ has the weak $\mathrm{P}$-property and $\mathrm{T}\left(\mathrm{A}_{0}\right) \subseteq \mathrm{B}_{0}$. Then there exists a unique $x^{*} \in A$ such that $\mathrm{d}^{*}\left(\mathrm{x}^{*}, \mathrm{~T} x^{*}\right)=\mathrm{d}^{*}(\mathrm{~A}, \mathrm{~B})$.

If we choose others $\psi, \phi$ in Example 1.5, by Theorem 3.2, we can get the relatively result. We omit it here.

\section{Acknowledgment}

This project is supported by the National Natural Science Foundation of China under grant (11071279).

\section{References}

[1] R. P. Agarwal, E. Karapınar, B. Samet, An essential remark on fixed point results on multiplicative metric spaces, Fixed Point Theory Appl., 2016 (2016), 3 pages. 1, 1, 1.3

[2] A. E. Bashirov, E. M. Kurplnar, A. Ozyaplcl, Multiplicative calculus and its applications, J. Math. Anal. Appl., 337 (2008), 36-48. 1, 1.1

[3] A. E. Bashirov, E. Misirli, Y. Tandogdu, A. Ozyaplcl, On modeling with multiplicative differential equations, Appl. Math. J. Chinese Univ., 26 (2011), 425-438. 1

[4] S. Czerwik, Contraction mappings in b-metric spaces, Acta Math. Inform. Univ. Ostraviensis, 1 (1993), 5-11. 1, 1.2

[5] K. Fan, Extensions of two fixed point theorems of F. E. Browder, Math. Z., 112 (1969), 234-240. 1, 1

[6] L. Florack, H. Van Assen, Multiplicative calculus in biomedical image analysis, J. Math. Imaging Vision, 42 (2012), 64-75.

[7] W. A. Kirk, S. Reich, P. Veeramani, Proximinal retracts and best proximity pair theorems, Numer. Funct. Anal. Optim., 24 (2003), 851-862. 1

[8] Y. Su, J.-C. Yao, Further generalized contraction mapping principle and best proximity theorem in metric spaces, Fixed Point Theory Appl., 2015 (2015), 13 pages. 1, 1.4, 1.5, 1.9, 1, 1.10, 1.11

[9] J. Zhang, Y. Su, Q. Cheng, A note on 'A best proximity point theorem for Geraghty-contractions', Fixed Point Theory Appl., 2013 (2013), 4 pages. 1, 1.6, 1.7, 1.8

[10] J. Zhang, Y. Su, Q. Cheng, Best proximity point theorems for generalized contractions in partially ordered metric spaces, Fixed Point Theory Appl., 2013 (2013), 7 pages. 1, 1.6, 1.7 